\title{
Coherent spin dynamics of an interwell excitonic gas in GaAs/AIGaAs coupled quantum wells
}

\author{
Larionov, A. V.; Bisti, V. E.; Bayer, M.; Hvam, Jørn Märcher; Sørensen, K.
}

Published in:

Physical Review B Condensed Matter

Link to article, DOI:

10.1103/PhysRevB.73.235329

Publication date:

2006

Document Version

Publisher's PDF, also known as Version of record

Link back to DTU Orbit

Citation (APA):

Larionov, A. V., Bisti, V. E., Bayer, M., Hvam, J. M., \& Sørensen, K. (2006). Coherent spin dynamics of an interwell excitonic gas in GaAs/AlGaAs coupled quantum wells. Physical Review B Condensed Matter, 73(23), 235329. https://doi.org/10.1103/PhysRevB.73.235329

\section{General rights}

Copyright and moral rights for the publications made accessible in the public portal are retained by the authors and/or other copyright owners and it is a condition of accessing publications that users recognise and abide by the legal requirements associated with these rights.

- Users may download and print one copy of any publication from the public portal for the purpose of private study or research.

- You may not further distribute the material or use it for any profit-making activity or commercial gain

- You may freely distribute the URL identifying the publication in the public portal 


\title{
Coherent spin dynamics of an interwell excitonic gas in GaAs/AlGaAs coupled quantum wells
}

\author{
A. V. Larionov and V. E. Bisti* \\ Institute of Solid State Physics, Russian Academy of Sciences, 142432, Chernogolovka, Russia \\ M. Bayer \\ Experimentelle Physik II, Universität Dortmund, D-44221 Dortmund, Germany \\ J. Hvam and K. Soerensen \\ Microelectronic Centret, Technical University of Denmark, DK 2800 Lyngby, Denmark \\ (Received 12 December 2005; revised manuscript received 9 May 2006; published 15 June 2006)
}

\begin{abstract}
The spin dynamics of an interwell exciton gas has been investigated in $n-i-n$ GaAs/AlGaAs coupled quantum wells. The time evolution kinetics of the interwell exciton photoluminescence has been measured under resonant excitation of the $1 s$ heavy-hole intrawell exciton, using a pulsed tunable laser. The formation of a collective exciton phase in time and the temperature dependence of its spin relaxation rate have been studied. The spin relaxation rate of the interwell excitons is strongly reduced in the collective phase. This observation provides evidence for the coherence of the interwell exciton collective phase at temperatures below a critical $T_{c}$. A theoretical analysis of the interwell exciton spin-flip dynamics has been developed.
\end{abstract}

DOI: 10.1103/PhysRevB.73.235329

PACS number(s): 73.21.Fg, 78.47.+p, 78.67.De, 78.66.Fd

\section{INTRODUCTION}

Among the quasi-two-dimensional systems based on semiconductor heterostructures, coupled quantum wells are of particular interest because they may provide a spatial separation of photoexcited electrons and holes in neighboring quantum wells. ${ }^{1}$ For example, in $n-i-n$ type GaAs/AlGaAs coupled quantum wells (CQWs) with tilted bands due to bias application, excitons can be excited with electron and hole confined in adjacent wells which are separated by a tunneling barrier. These excitons are called spatially indirect or interwell excitons (IEs), different from the direct intrawell excitons (DEs), for which electron and hole are located in the same QW. In contrast to DEs, IEs are long lived because the wave functions of electron and hole overlap very weakly through the tunneling barrier. A large number of IEs can therefore easily be accumulated and this exciton gas can be cooled down to rather low temperatures. Various possible scenarios of collective behavior of a dense system of spatially separated electrons and holes have been considered theoretically. ${ }^{1-3}$ Further, there are already quite a few reports on collective behavior of IEs upon reaching critical conditions (see Refs. 4-6).

Earlier, we found that below a critical temperature the gas of IEs in CQWs undergoes a phase-transition-like behavior with increasing exciton density. ${ }^{7}$ Experimental findings such as strong narrowing of the IEs photoluminescence (PL) line, drastic increasing of its circular polarization degree, and high sensitivity with respect to temperature have been associated with the formation of an IE collective dielectric phase. Later it was shown that if critical conditions are satisfied the IE collective phase is most likely to occur in domain regions with lateral confinement. ${ }^{8}$ According to our experiments the condensation occurs at $T<4 \mathrm{~K}$ for an average exciton concentration of $n_{e x} \sim 3 \times 10^{10} \mathrm{~cm}^{-2}$.

A collective excitonic phase, corresponding to a macroscopic exciton occupation of the energetically lowest state in the domain, should show spatial and temporal coherence.
This means that within the coherence length condensed excitons are described by a joint wave function. Consequences are not only an increase of the radiative decay rate of the excitons, but also a reduction of the exciton spin relaxation rate. Due to these features the opportunity for photoexcitation of a spin-aligned collective IE phase might arise. In the considered case the coherence length scale is expected to be equal to the size of the localization domain, arising from long-range potential fluctuations (around $1 \mu \mathrm{m}$ in lateral size), in which IEs can be accumulated.

In the present paper we address in detail the IE spin dynamics by analyzing the circular polarization degree of their PL after resonant pulsed laser photoexcitation. In addition to our previous work, ${ }^{7}$ we have observed a further peculiarity which is a strong argument for the coherence of the dense IE gas, namely, a strong reduction of the spin relaxation rate upon reaching critical conditions. We also provide a theoretical modeling for the IE spin flip at low temperatures.

The paper is organized as follows. After describing the CQW heterostructures being studied as well as the experimental technique in Sec. II A, we describe in Sec. II B the time evolution of the IE luminescence under conditions of resonant pulsed laser excitation. A broad set of experimental parameters (pump power, electrical bias, and temperature) was varied. In Sec. III we discuss the theoretical analysis of the IE spin relaxation rate. Finally, in the concluding Sec. IV the spin relaxation data are discussed in relation to various other properties of IEs observed in their luminescence spectra. In particular, their critical behavior as function of optical pumping and temperature is interpreted in terms of a collective behavior.

\section{EXPERIMENT}

\section{A. Samples and experimental setup}

We have investigated an $n-i-n$ GaAs/AlGaAs heterostructure containing a GaAs/AlAs/GaAs CQW structure with a 


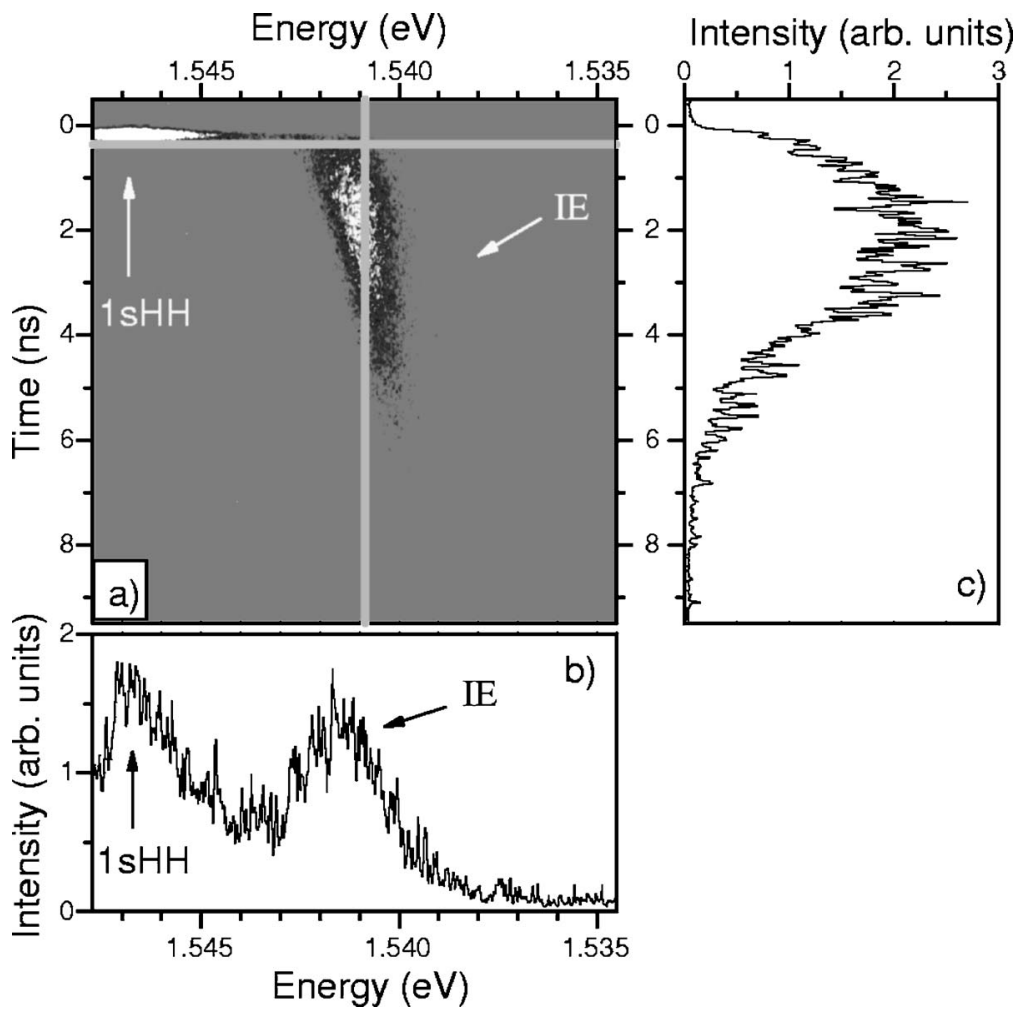

FIG. 1. (a) Streak camera image of the PL signal as result of excitation of the sample by a short circularly polarized $\left(\sigma^{+}\right)$laser pulse (about 1 ps duration). The brightness corresponds to the PL intensity. The horizontal axis gives energy, the vertical axis gives time. (b) Horizontal cut through the image [as indicated by the corresponding line in (a)] gives the PL spectrum at a fixed time. (c) Vertical cut [as shown by the vertical line in (a)] gives the PL kinetics at a fixed wavelength. Arrows indicate signal from the DE $(1 s \mathrm{HH})$ and the IE. The image was obtained for a bias voltage $U=0.65 \mathrm{~V}$ at $T=1.85 \mathrm{~K}$ and an excitation power of about $30 \mathrm{~kW} / \mathrm{cm}^{2}$.

width of the GaAs wells of about $120 \AA$, and a width of the AlAs barrier of about $11 \AA$. For details see Reference 3 . The IEs PL was excited by 120 fs laser pulses with a repetition rate of $76 \mathrm{MHz}$. A holographic grating with optical slits has been used for pulse shaping. The detection of the signal was provided by a Hamamatsu streak camera (Model 5680-24) with a $\mathrm{Si}$ charged-coupled device detector attached to a $0.5 \mathrm{~m}$ spectrometer (Acton $\mathrm{SP}-500 \mathrm{i}$ ). The system time resolution was about 70 ps. For circular polarization analysis of the PL signal under resonant photoexcitation we have used linear polarizers and a quarter-wave retarder.

\section{B. Experimental results}

Figure 1(a) gives a contour plot of a streak camera image of the PL emission from the studied CQWs. The horizontal (vertical) axis gives energy (time), while the brightness gives the PL intensity. For data analysis this image has been profiled either along the energy axis [Fig. 1(b)] or along the time axis [Fig. 1(c)], resulting in time-resolved PL spectra and energy-resolved PL decay curves, respectively. For spin orientation of the IEs we used circularly polarized (for example, $\left.\sigma^{+}\right)$laser excitation resonant to the $1 s$ heavy-hole $(\mathrm{HH})$ ground state of the DEs. The IE PL kinetics was measured under these conditions for different temperatures and bias voltages.

Earlier we had studied already the time evolution of PL spectra. ${ }^{7}$ It was found that shortly after zero delay, the IE PL is strongly circularly polarized (more than 60\%), following the polarization of the exciting laser, and has a full width at half maximum of about $3 \mathrm{meV}$. As the delay increases the PL line narrows and shifts toward the long-wavelength part of the spectrum. Within the first nanosecond after the laser pulse, the IE PL line remains strongly circularly polarized without any strong variations across the whole spectrum. At later times only the high-energy part of the spectrum remains polarized. For low temperature $T=2 \mathrm{~K}$ the circular polarization of the PL disappears after $7 \mathrm{~ns}$ while at $T$ above $6 \mathrm{~K}$ the $\mathrm{PL}$ is unpolarized already after $3 \mathrm{~ns}$.

In the present work we will concentrate on a detailed investigation of the IE spin flip for varying temperatures. For a quantitative analysis of the IE spin relaxation, the PL image is profiled along the time axis, from which PL decay curves for a fixed energy interval of $1 \mathrm{meV}$ width are obtained. Figure 2 gives the IE PL decay curves for $\sigma^{+}$and $\sigma^{-}$circular polarization (circular and square symbols, respectively) as well as the decay of the corresponding circular polarization degree (triangular symbols), measured near the PL line maximum at different temperatures [vertical gray line in Fig. 1(a)]. The maximum intensity of the IE PL line is reached for delays of about 2 ns. We suggest that this time is necessary for the formation of IEs upon resonant tunneling mainly of electrons to the adjacent quantum well and relaxation in energy. We want to emphasize that qualitatively the same results are obtained for integration at different center energies along the PL trace.

The spin relaxation time is the decay time of the circular polarization degree. The circular polarization is defined as $\gamma=\left(I_{\sigma^{+}}-I_{\sigma^{-}}\right) /\left(I_{\sigma^{+}}+I_{\sigma^{-}}\right)$, where $I_{\sigma^{+}, \sigma^{-}}$are the PL intensities for $\sigma^{+}$and $\sigma^{-}$polarization, respectively. From Fig. 2 it is evident that $\gamma$ strongly depends on temperature.

We find that the spin relaxation dynamics can be described by two time constants, an initial fast one and a delayed slow one (solid lines in Fig. 2 give corresponding least-mean-square fits to the data using a biexponential decay form). The initial decay time $\tau_{1}$ is very weakly temperature 


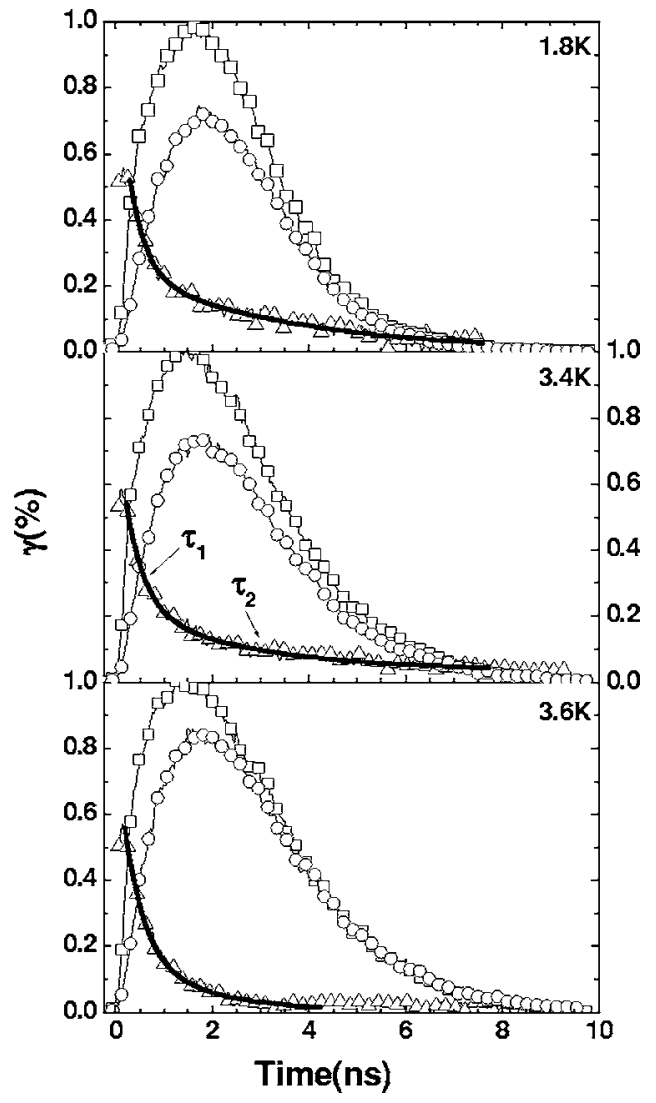

FIG. 2. Circular polarization resolved decay curves of the IE PL $\left(\sigma^{+}\right.$, squares; $\sigma^{-}$, circles; corresponding to detection co- and crosspolarized with the laser excitation, respectively) and its circular polarization degree (triangles) measured at different temperatures within the vertical gray line in Fig. 1, corresponding to a spectral window of $1 \mathrm{meV}$ for $U=0.6 \mathrm{~V}$ and an excitation power of about $30 \mathrm{~kW} / \mathrm{cm}^{2}$. Solid curves are biexponential fits to the circular polarization degree.

dependent and amounts to about 0.35 ns (see Fig. 3). In contrast, the slow decay time $\tau_{2}$ which exceeds $\tau_{1}$ by an order of magnitude at low $T$ drops by a factor of about 2 for temperatures above $3.6 \mathrm{~K}$. Up to $15 \mathrm{~K}$ no more significant change in the temporal dynamics of the IE circular polarization degree is observed. The same behavior occurs for another, slightly smaller bias $U=-0.55 \mathrm{~V}$ applied to the CQWs. These data have been recorded at an excitation power of about $30 \mathrm{~kW} / \mathrm{cm}^{2}$ leading to an IE concentration $n_{e x} \sim 3$ $\times 10^{10} \mathrm{~cm}^{-2}$. We have found that the strong reduction of spin relaxation time $\tau_{2}$ is quite sensitive to excitation power (see Fig. 4). At smaller and at bigger excitation power the temperature boundary for the strong reduction of the spin relaxation time $\tau_{2}$ shifts to lower temperatures. Note that the biexponential spin-flip decay is observed in a rather narrow IE concentration range: $n_{e x} \sim 10^{10}-10^{11} \mathrm{~cm}^{-2}$.

Figure 5 shows the bias dependence of the spin relaxation time at $T=2 \mathrm{~K}$. With increasing voltage up to about $0.6 \mathrm{~V}, \tau_{1}$ and $\tau_{2}$ first monotonically increase and then do not change up to $0.85 \mathrm{~V}$, corresponding to about $22 \mathrm{meV}$ splitting between the $1 s \mathrm{HH}$ and the IE. For higher bias, the spin relaxation process is described by a single relaxation time. From Fig. 5 it can be seen that with increasing bias the time for

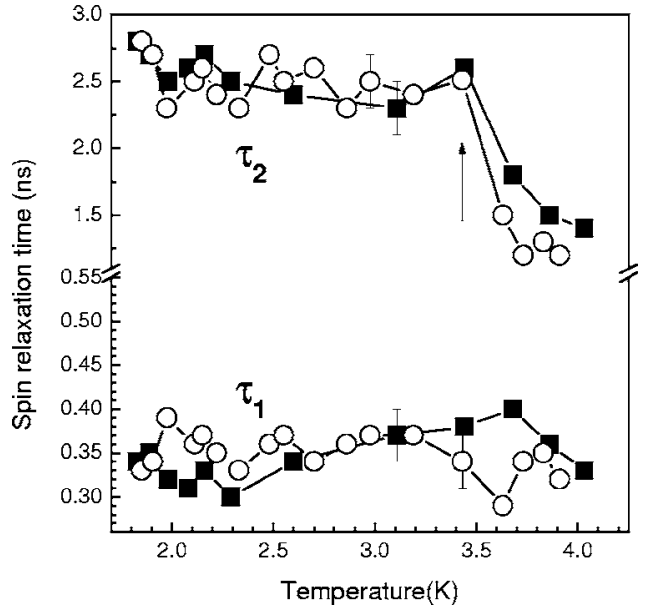

FIG. 3. Temperature dependence of spin relaxation times. Note the break in the $y$ axis. The circles give the fast, $\tau_{1}$, and the slow, $\tau_{2}$, relaxation times at bias $U=0.6 \mathrm{~V}$, and the squares give the $\tau_{1}$ and $\tau_{2}$ times at $U=0.55 \mathrm{~V}$, both at an excitation power of about $30 \mathrm{~kW} / \mathrm{cm}^{2}$. The arrow indicates the temperature where a strong change of the spin relaxation rate $\tau_{2}^{-1}$ occurs.

accumulation of IEs increases. This can be attributed to a rise of the tunneling time. Therefore we suggest that for large bias single-particle spin relaxation mechanisms may play a determinative role, causing a mono exponential decay.

We have also measured the temperature dependence of the circular polarization degree of the intrawell $1 s \mathrm{HH}$ exciton luminescence. Its spin relaxation rate does not change in the temperature interval from 2 to $15 \mathrm{~K}$ and is described by a monoexponential decay with a time constant of about 180 ps. This result is in good agreement with Ref. 11, where the exciton spin relaxation dynamics has been investigated in great detail at low temperatures. The mechanism responsible

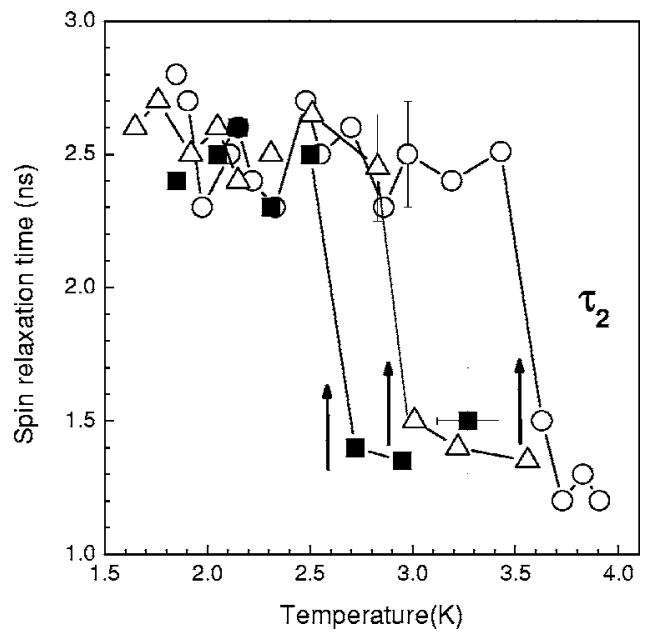

FIG. 4. Temperature dependence of the spin relaxation time $\tau_{2}$ at different excitation powers and at $U=0.6 \mathrm{~V}$. Open circles correspond to an excitation power of about $30 \mathrm{~kW} / \mathrm{cm}^{2}$ leading to an IE concentration $n_{e x} \sim 3 \times 10^{10} \mathrm{~cm}^{-2}$. Squares and triangles correspond to excitation powers of about 20 and $45 \mathrm{~kW} / \mathrm{cm}^{2}$, respectively. The arrows indicate the temperature region where a strong change of the spin relaxation rate occurs. Lines are guides to the eye. 


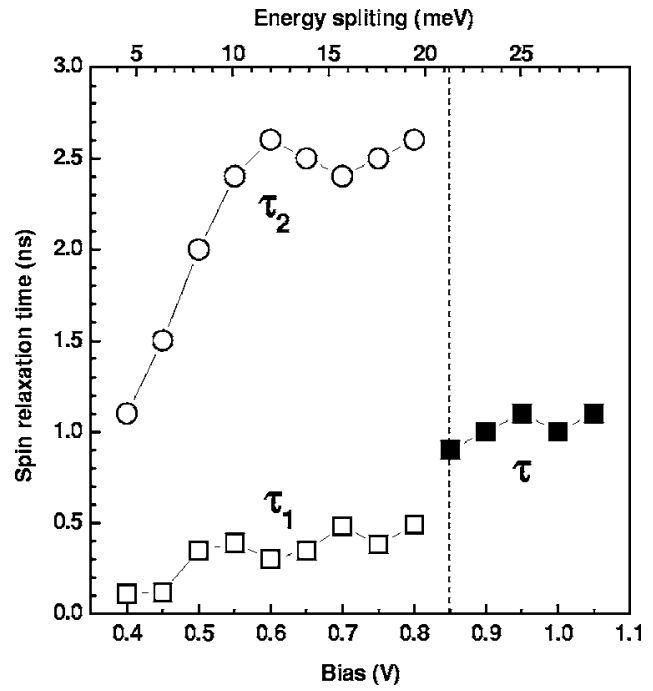

FIG. 5. Bias dependencies of the spin relaxation times at $T$ $=2 \mathrm{~K}$ and an excitation power of about $30 \mathrm{~kW} / \mathrm{cm}^{2}$. Circles correspond to the slow $\tau_{2}$ relaxation times, open squares to the fast $\tau_{1}$. Full squares give the spin relaxation times $\tau$ for high bias where a monoexponential decay of the circular polarization degree is observed. The dashed line indicates the boundary between the biexponential and the monoexponential spin relaxation ranges.

for $1 s \mathrm{HH}$ exciton spin relaxation is the electron-hole exchange interaction. For CQW structures the carrier spin dynamics is more complicated. After the laser pulse several processes may occur: electron tunneling to the adjacent quantum well, energy and spin relaxation, IE formation, and radiative annihilation. We suggest that the $\tau_{1}$ time is due to electron-hole exchange interaction within the $1 s \mathrm{HH}$ exciton, while the $\tau_{2}$ time characterizes the IE spin relaxation. Since the wave function overlap integral is very small, the electron-hole exchange interaction is also weak and the $\tau_{2}$ time is much longer than the $\tau_{1}$ time.

\section{THEORETICAL DESCRIPTION OF IE SPIN RELAXATION}

The exciton kinetics including spin relaxation is governed by the following processes: (1) electron spin flip within the exciton with rate $w_{e}$, (2) hole spin flip within the exciton with rate $w_{h}$, (3) exciton spin flip due to electron-hole exchange with rate $w_{E X}$ for DEs and with rate $w_{e x}$ for IEs, (4) exciton radiative recombination with rate $w_{R}$ for DEs and with rate $w_{r}$ for IEs, and (5) DE transformation into IEs due to electron tunneling to the adjacent quantum well with rate $w_{k}$.

For modeling, we have used the equations derived in Refs. 10 and 11. The concentrations of DEs, $N_{i}^{D}$, and IEs, $N_{i}^{I}$, are described by the rate equations

$$
\begin{gathered}
\frac{d}{d t} N_{i}^{D}=F_{i j}^{D} N_{j}^{D}-w_{k} N_{i}^{D}, \\
\frac{d}{d t} N_{i}^{I}=F_{i j}^{I} N_{j}^{I}+w_{k} N_{i}^{D},
\end{gathered}
$$

where the indices $i, j=2,1,-1,-2$ give the projection of the exciton angular momentum onto the spin quantization axis defined by the heterostructure growth direction. The exciton angular momentum is the sum of the electron spin and the hole angular momentum. States $| \pm 1\rangle$ corresponding to $i, j$ $=1,-1$ are optically active ("bright"), whereas states $| \pm 2\rangle$ corresponding to $i, j=2,-2$ are optically inactive states ("dark").

These equations have to be solved for the boundary conditions

$$
N_{1}^{D}=N_{0}, \quad N_{i}^{D}=0, \quad i \neq 1, \quad N_{i}^{I}=0 .
$$

The coefficients $F_{i j}^{D / I}$ are given by

$$
F_{i j}^{D(I)}=\left|\begin{array}{cccc}
-\left(w_{e}^{+}+w_{h}^{+}\right) & w_{e}^{-} & w_{h}^{-} & 0 \\
w_{e}^{+} & W^{D(I)} & w_{E X(e x)} & w_{h}^{+} \\
w_{h}^{+} & w_{E X(e x)} & W^{D(I)} & w_{e}^{+} \\
0 & w_{h}^{-} & w_{e}^{-} & -\left(w_{e}^{+}+w_{h}^{+}\right)
\end{array}\right| .
$$

The DE and IE spin-flip probabilities are

$$
W^{D(I)}=-\left(\frac{1}{\tau_{R(r)}}+w_{E X(e x)}+w_{e}^{-}+w_{h}^{-}\right) .
$$

The electron (hole) spin-flip rate inside the exciton is given by

$$
w_{e(h)}^{ \pm}=-\frac{w_{e(h)}}{1+e^{ \pm \Delta / k T}},
$$

where $\Delta>0$ is the energy splitting between the optically active and the optically inactive states.

We assume that the hole spin flip time is much shorter than all other times in our system. In this case the bright $| \pm 1\rangle$ and dark $|\mp 2\rangle$ exciton concentrations are connected to each other, depending on temperature $T$ and exchange splitting $\Delta$,

$$
N^{ \pm 2}=N^{\mp 1} f(\Delta / k T), \quad f=\frac{1+e^{\Delta / k T}}{1+e^{-\Delta / k T}} .
$$

This assumption allows one to take into consideration only the bright excitons,

$$
\frac{d}{d t} N_{i}^{D}=G_{i j}^{D} N_{j}^{D}
$$

with

$$
\begin{gathered}
N_{1}^{D}=N_{0}, \quad N_{i}^{D}=0, \quad i= \pm 1, \\
\frac{d}{d t} N_{i}^{I}=G_{i j}^{I} N_{j}^{I}+w_{k} N_{i}^{D},
\end{gathered}
$$

with $N_{i}^{I}=0$. The coefficients $G_{i j}^{D(I)}$ are given by

$$
G_{i j}^{D(I)}=\left|\begin{array}{cc}
-\left(w_{L(l)}+w_{X(x)}\right) & w_{X(x)} \\
w_{X(x)} & -\left(w_{L(l)}+w_{X(x)}\right)
\end{array}\right| .
$$

The influence of the dark $| \pm 2\rangle$ excitons is comprised in the effective annihilation rates $w_{L}, w_{l}$ and the spin relaxation rates $w_{X}, w_{x}$ (effective times $\tau_{L}, \tau_{l}$ and $\tau_{X}, \tau_{x}$ ) for DEs and IEs, respectively, 


$$
\begin{gathered}
w_{L}=w_{k}+w_{R} /(1+f), \\
w_{l}=w_{r} /(1+f), \\
w_{X}=2 w_{E X} /(1+f)+4 w_{e} /\left(2+e^{\Delta / k T}+e^{-\Delta / k T}\right), \\
w_{x}=2 w_{e x} /(1+f)+4 w_{e} /\left(2+e^{\Delta / k T}+e^{-\Delta / k T}\right) .
\end{gathered}
$$

To compare these results with the experimental data it is necessary to determine the time dependence of the exciton concentration of the bright states and of their spin polarization degree:

$$
\begin{gathered}
N_{ \pm 1}(t)=\frac{1}{2} N(t)[1 \pm p(t)], \\
p(t)=\frac{N_{+1}(t)-N_{-1}(t)}{N_{+1}(t)+N_{-1}(t)},
\end{gathered}
$$

where $N(t)=N_{+1}(t)+N_{-1}(t)$ is the total exciton concentration. The IE PL intensities measured in experiment are directly proportional to the exciton concentrations. For the DEs we obtain

$$
N^{D}(t)=N_{0} e^{-w_{L} t}, \quad p^{D}(t)=e^{-w_{X} t},
$$

while for the IEs we have

$$
\begin{gathered}
N^{I}(t)=\frac{N_{0} w_{k}}{w_{L}-w_{l}}\left(e^{-w_{l} t}-e^{-w_{L} t}\right), \\
p^{I}(t)=\frac{w_{L}-w_{l}}{w_{L}+w_{X}-w_{l}-w_{x}} \frac{e^{-\left(w_{l}+w_{x}\right) t}-e^{-\left(w_{L}+w_{X}\right) t}}{e^{-w_{l} t}-e^{-w_{L} t}} .
\end{gathered}
$$

Comparison of experimental data with theoretical calculations is presented in Fig. 6 for different electrical biases corresponding to the following energy splittings $\Delta E$ between the $1 s \mathrm{HH}$ exciton and the IE:

$$
\begin{gathered}
\text { (2) } \Delta E=6.4 \mathrm{meV}, \quad \tau_{L}=0.20 \mathrm{~ns}, \quad \tau_{l}=1.0 \mathrm{~ns}, \\
\tau_{X}=0.13 \mathrm{~ns}, \quad \tau_{x}=1.6 \mathrm{~ns}, \\
\text { (3) } \Delta E=8.3 \mathrm{meV}, \quad \tau_{L}=0.27 \mathrm{~ns}, \quad \tau_{l}=1.3 \mathrm{~ns}, \\
\tau_{X}=0.16 \mathrm{~ns}, \quad \tau_{x}=2.0 \mathrm{~ns} .
\end{gathered}
$$

Our model rather well describes the spin relaxation at small bias, while at large voltages there are some discrepancies. Probably in this regime nonlocal tunneling would have to be taken into account for better agreement.

The increase of the exciton spin relaxation time and the radiative exciton annihilation time with bias can be explained by the enhanced spatial separation of electrons and holes both in the same quantum well as well as in adjacent ones.

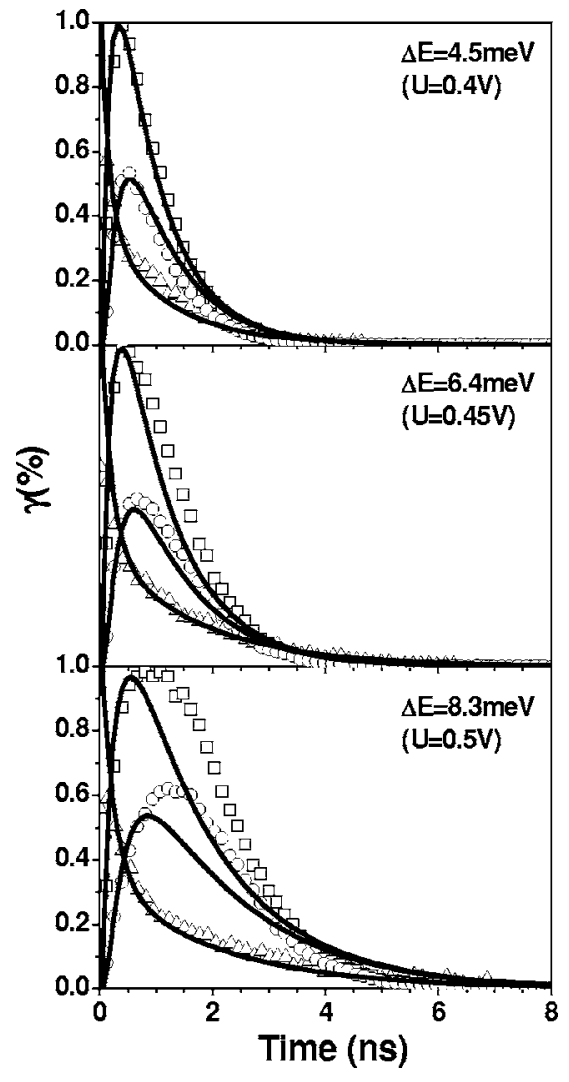

FIG. 6. Circularly polarized IE PL decay curves $\left(\sigma^{+}\right.$, squares; $\sigma^{-}$, circles) as well as the corresponding circular polarization degree $\gamma$ (triangles) measured at different biases. The signal has been integrated over the vertical gray line in Fig. 1 covering an energy window of $1 \mathrm{meV}$. Solid curves correspond to theoretical calculations according to expressions (14) and (15). $T=2 \mathrm{~K}$.

\section{DISCUSSION}

We believe that the experimental results on the change of the IE spin relaxation described above are due to the collective behavior of the IEs below a critical temperature. ${ }^{7}$ Our claim is based on Ref. 12 where the spin relaxation rate of Bose condensates of atoms in traps was studied. It has been shown that the spin relaxation rate of the atoms condensed phase is $N$ ! times smaller than that of atoms in the uncondensed phase, where $N$ is the number of particles involved in a scattering process destroying Bose condensation. Experimentally, this claim was confirmed in Ref. 13, in which the spin dynamics of atoms in a Bose-condensate has been investigated. In our case the $\tau_{2}$ time characterizing the exciton spin relaxation changes by a factor of about 2 , as expected from the electron-hole composition of the exciton, in good agreement with the model calculations in Ref. 12.

This finding is another indication for the IE collective coherence upon reaching critical conditions. Qualitatively, the time evolution of this phase can be described as follows. At small delays $(<1 \mathrm{~ns})$, the IE PL line is rather broad due to the fluctuating potential relief in the quantum-well plane. The potential traps arise, for example, from interface roughnesses or other structural imperfections. This is manifested by the narrowing of the PL line with increasing delay, when 
more and more IEs form. Then the linewidth ceases to reflect the statistical distribution of the fluctuation amplitudes of the random potential. In our opinion, the sharp narrowing of the PL line, the superlinear rise of its intensity, and the lowenergy shift of the PL line observed for our samples cannot be associated with reaching the percolation threshold by the IE density only, because of the strong sensitivity to temperature, even though there is no distinct temperature boundary. Berman and Lozovik have shown ${ }^{2}$ that a sufficiently dense system of IEs with particular values of the dipole moment may condense into a dielectric collective phase despite of the dipole repulsion between such excitons.

A consequence of the collective behavior of the IEs is its coherency. This means that the IEs possess the same phase on the length scale of the de Broglie wavelength, which is close to the lateral domain size. ${ }^{8}$ This phase coherence in turn affects the radiative annihilation rate, which increases due to the increased coherence volume. Earlier we have found from the kinetics of luminescence spectra that the lifetime of the collective exciton state is about three times shorter than the luminescence decay time of localized IEs. ${ }^{9}$ This increase in the radiative decay rate of IEs and the corresponding increase of the degree of circular polarization are particular manifestations of the coherence of the collective exciton state. Another consequence of the IE coherence is the essential increase of the IE spin-flip time at temperatures below the critical one. As found in previous investigations, the analog of a phase diagram can be constructed for the collective exciton state. ${ }^{14}$ According to it, $T_{c}$ increases with increasing IE concentration, but there is both an upper and a lower boundary for the existence of the collective phase. In the present study we have observed that close to these IE concentration boundaries the spin-flip time drops at smaller temperatures, in good qualitative agreement with Ref. 14.

\section{CONCLUSIONS}

In conclusion, the temperature dependence of the IE spin relaxation time has been investigated in GaAs/AlGaAs CQWs. A strong decrease of the relaxation rate has been discovered at $T<T_{c}=4 \mathrm{~K}$. The observed phenomenon occurs due to the IE phase coherence at temperatures below the critical one. A theoretical model of the IE spin flip has been developed.

\section{ACKNOWLEDGMENTS}

The authors want to express their thanks to V. B. Timofeev for valuable suggestions and remarks, as well as to Yu. Kagan for fruitful and interesting discussions. This research was supported by DFG (Grant No. 436 RUS 17/95/03), by RFBR, and by the Russian Science Support Foundation.
*Electronic address: larionov@issp.ac.ru

${ }^{1}$ Xuejun Zhu, P. L. Littlewood, M. S. Hybersten, and T. Rice, Phys. Rev. Lett. 74, 1633 (1995).

${ }^{2}$ Yu. E. Lozovik and O. L. Berman, JETP 84, 1027 (1997).

${ }^{3}$ A. V. Larionov and V. B. Timofeev, JETP 90, 1093 (2000).

${ }^{4}$ L. V. Butov, J. Phys.: Condens. Matter 16, R1577 (2004).

${ }^{5}$ S. A. Moskalenko and D. W. Snoke, Bose-Einstein Condensation of Excitons and Biexcitons (Cambridge University Press, Cambridge, U.K., 2000).

${ }^{6}$ A. V. Larionov and V. B. Timofeev, JETP Lett. 73, 301 (2001).

${ }^{7}$ A. V. Larionov, V. B. Timofeev, J. Hvam, and C. Soerensen, JETP Lett. 71, 117 (2000).

${ }^{8}$ A. V. Larionov, V. B. Timofeev, P. A. Ni, S. V. Dubonos, J. Hvam, and C. Soerensen, JETP Lett. 75, 570 (2002).
${ }^{9}$ A. V. Larionov, V. B. Timofeev, J. Hvam, and C. Soerensen, JETP Lett. 75, 200 (2002).

${ }^{10}$ M. Z. Maialle, E. A. de Andrada e Silva, and L. J. Sham, Phys. Rev. B 47, 15776 (1993).

${ }^{11}$ A. Vinattieri, Jagdeep Shah, T. C. Damen, D. S. Kim, L. N. Pfeiffer, M. Z. Maialle, and L. J. Sham, Phys. Rev. B 50, 10868 (1994).

${ }^{12}$ Yu. Kagan, V. A. Kashurnikov, A. V. Krasavin, N. V. Prokof'ev, and B. V. Svistunov, Phys. Rev. A 61, 043608 (2000).

${ }^{13}$ E. A. Burt, R. W. Ghrist, C. J. Myatt, M. J. Holland, E. A. Cornell, and C. E. Wieman, Phys. Rev. Lett. 79, 337 (1997).

${ }^{14}$ A. A. Dremin, V. B. Timofeev, A. V. Larionov, J. Hvam, and C. Soerensen, JETP Lett. 76, 450 (2002). 